\title{
Modelación de curvas de lactancia para producción de leche, grasa y proteína en bovinos Holstein en Antioquia, Colombia
}

\author{
Modeling the lactation curves for milk, fat and protein yield of \\ Holstein cattle in Antioquia, Colombia
}

\author{
Jhon Cañas A, ${ }^{1}$ M.Sc, Mario Cerón-Muñoz, ${ }^{1}, 2 *$ Ph.D, Juan Corrales $A,{ }^{1}$ M.Sc.
}

\begin{abstract}
${ }^{1}$ Universidad de Antioquia, Facultad de Ciencias Agrarias e Instituto de Biología, GaMMA: Grupo de investigación en Genética, Mejoramiento y Modelación Animal, Medellín, Colombia. ${ }^{2}$ Universidad de Antioquia, Facultad de Ciencias Agrarias, Medellín, Colombia. *Correspondencia: mceronm@agronica.udea.edu.co
\end{abstract}

Recibido: Junio de 2010; Aceptado: Febrero de 2011.

\section{RESUMEN}

Objetivo. Modelar curvas de lactancia para producción de leche y para los porcentajes de grasa y proteína y determinar los principales factores que influyen en la curva de lactancia. Materiales y métodos. Fueron empleados los datos de control lechero de 1532 animales de raza Holstein pertenecientes a 19 haciendas ubicadas en el departamento de Antioquia, Colombia, donde se compararon los modelos matemáticos no lineales propuestos por Wood, Brody, Papajcsik y Bodero y Wilmink. En el modelo que presentó los mejores ajustes se determinó el efecto del parto, la época de parto y el año de parto. Resultados. Las curvas de lactancia para producción de leche y porcentaje de grasa y proteína, estimadas por el modelo de Wood, presentaron los mejores valores en los criterios de comparación empleados. Los efectos de año y número de parto fueron significativos para los parámetros estimados en el modelo de Wood. Conclusiones. El modelo de Wood mostró el mejor ajuste en curvas de lactancia para producción de leche y para los porcentajes de grasa y proteína. La curva de lactancia para el porcentaje de proteína se caracterizó por ser muy consistente y no presentar mayores fluctuaciones durante toda la lactancia.

Palabras clave: Control lechero, época del parto, modelos matemáticos, tasa de partos. (Fuente: $C A B)$. 


\section{ABSTRACT}

Objective. Modeling lactation curves for milk yield and for fat (\%) and protein (\%) and to determine the main factors affecting the lactation curve. Materials and methods. Official milk control data of 1532 Holstein dairy cows from 19 farms from the department of Antioquia, Colombia were used. Non-linear mathematical models proposed by Wood, Brody, Papajcsik and Bodero and Wilmink were calculated. Regression coefficients of the best fit non linear model selected was used for the effect on parity, time and year of birth was. Results. Lactation curves for milk yield and both fat and protein percentages estimated by Wood's model showed the best values for the comparison criteria used. Parity and year of parity effects were significant for the estimated parameters in the Wood's model. Conclusions. Wood's model showed the best fit in lactation curves for milk yield and for fat (\%) and protein (\%). The lactation curve for protein (\%) was characterized by a very consistent production and no major fluctuations throughout lactation.

Key words: Calving rate, calving season, mathematical models, milk recording. (Source: $C A B)$.

\section{INTRODUCCIÓN}

En el mejoramiento de la calidad de la leche, tanto los factores genéticos como ambientales desempeñan un papel muy importante que debe ser reconocido y comprendido (1).

Al igual que la producción de leche, los porcentajes de grasa y proteína también pueden ser modelados empleando modelos probabilísticos $(2,3)$. Los modelos más empleados han sido los no lineales como el de gamma incompleto descrito por Wood (4), el modelo de Wilmink (5), Brody et al (6), entre otros.

La forma de la curva para los porcentajes de grasa y proteína siguen una relación inversa a la curva de producción de leche. Así, durante los primeros días correspondientes al calostro, los componentes sólidos en la leche son altos, pero caen rápidamente en la misma proporción en que la producción de leche incrementa; hacia el último tercio de la lactación el incremento de los sólidos vuelve a ser significativo (7).

Existen numerosos factores ambientales que influyen en la producción de leche, grasa y proteína y que consecuentemente alteran la forma de la curva de lactancia en ganado Holstein, entre ellos los más influyentes son el número de parto y la época y año de parto $(1,3,7)$.
El objetivo de este trabajo fue modelar curvas de lactancia para producción de leche y para los porcentajes de grasa y proteína y determinar los principales factores que influyen en la curva de lactancia.

\section{MATERIALES Y MÉTODOS}

Obtención de datos. Se emplearon los datos de control lechero mensual, realizado en los años de 2008 y 2009, de 19 haciendas ubicadas en el norte y el oriente del departamento de Antioquia en los municipios de San Pedro, Bello, Entrerrios, Belmira, Rionegro y La Ceja.

Variables climáticas. La temperatura media fue de $15^{\circ} \mathrm{C}$, con precipitaciones de 1570 mm/año, altitud de 2300 a 2500 msnm y humedad relativa del $72 \%$.

Meses del parto. Los meses de parto fueron agrupados por trimestres en cuatro épocas: diciembre-febrero, marzo-mayo, junio-agosto y septiembre-noviembre.

\section{Manejo de los animales y controles} lecheros. Los animales se encontraban bajo un sistema de pastoreo rotacional, en pasto Kikuyo (Pennisetum clandestinum). Las vacas fueron ordeñadas dos veces al día y recibían alimento concentrado entre 
6 y $12 \mathrm{Kg}$ dependiendo de la producción.

Para este análisis se contó con un total de 13640 datos de controles lecheros mensuales de 2156 lactancias de 1532 vacas Holstein (Tabla 1). Animales con lactancias inferiores a 100 días de producción y menos de cuatro controles lecheros por lactancia no fueron tenidos en cuenta para este análisis.

El control lechero consistió en mediciones individuales de leche, grasa y proteína mediante visitas mensuales en los dos ordeños (am y pm). Para lo anterior se emplearon equipos portátiles (Ekomilk) que permiten la valoración de los constituyentes sólidos de la leche.

\section{Obtención de muestras individuales}

de leche. Fueron obtenidas al finalizar el ordeño de cada animal a través del medidor de leche (ordeño en sala) o directamente del balde, previa homogenización de la muestra (ordeño en potrero). Estas muestras fueron almacenadas en tubos con capacidad de $50 \mathrm{ml}$ para ser analizadas por medio del analizador de leche portátil (Ekomilk) a un rango de temperatura de entre 20 y $28^{\circ} \mathrm{C}$ con homogenización previa y calentamiento al baño maría

Tabla 1. Distribución del número de controles lecheros mensuales y el número de animales empleados en este análisis.

\begin{tabular}{ccc}
\hline & $\begin{array}{c}\text { Controles } \\
\text { lecheros } \\
\text { mensuales }\end{array}$ & $\begin{array}{c}\text { Número de } \\
\text { animales }\end{array}$ \\
\hline Parto & 2974 & 395 \\
1 & 2980 & 329 \\
2 & 2465 & 241 \\
3 & 1907 & 210 \\
4 & 1545 & 174 \\
5 & 1769 & 183 \\
$\geq 6$ & & \\
Año de Parto & 2083 & 372 \\
2007 & 8959 & 802 \\
2008 & 2598 & 358 \\
2009 & & \\
\hline
\end{tabular}

según las especificaciones del equipo. Los equipos fueron calibrados continuamente por medio de pruebas de laboratorio.

Modelación de curvas de lactancia. Para cada animal en cada lactancia se evaluaron los siguientes modelos matemáticos no lineales:

Wood (4): $y_{i}=\beta_{0} t_{i}{ }^{\beta_{1}} e^{\left(-\beta_{2} t_{i}\right)}+e_{i}$

Brody et al (6): $y_{i}=\beta_{0} e^{\left(-\beta_{1} t_{i}\right)}-\beta_{0} e^{\left(-\beta_{2} t_{i}\right)}+e_{i}$

Wilmink (5): $y_{i}=\beta_{0}+\beta_{1} t_{i}+\beta_{2} e^{\left(-0.05 t_{i}\right)}+e_{i}$

Papajcsik \& Bodero (8): $y_{i}=\beta_{0} t_{i} e^{\left(-\beta_{2} t_{i}\right)}+e_{i}$

Donde, $y_{i}$ es la producción de leche, porcentaje de grasa o porcentaje de proteína a un tiempo $(i)$ determinado, los $\beta_{0}, \beta_{1}$ y $\beta_{2}$ son los parámetros a estimar en la función, e es la función exponencial, $t$ son los días en leche y $e_{i}$ es el error asociado a cada observación.

Para la elaboración de las curvas se utilizó el procedimiento NLIN del paquete estadístico SAS $®(9)$, donde se empleó el método de iteracción de Gauss-Newton.

Para la elección del modelo que mejor ajustó las curvas de lactancia se tuvo en cuenta el porcentaje de curvas que convergieron (PCCON), el porcentaje de curvas significativas (PCSIG) $(p<0.05)$, los criterios de información de Akaike (AIC) y Bayesiano (BIC), el porcentaje de curvas con el valor más bajo de AIC (PAIC) y de BIC (PBIC), los valores del coeficiente de determinación $\left(R^{2}\right)$ y el porcentaje de curvas con el valor más alto de $\mathrm{R}^{2}\left(\mathrm{PR}^{2}\right)$.

Para el modelo que presentó los mejores ajustes se estimó por medio de la primera derivada el tiempo en el cual se alcanza el pico de producción $\left(T_{\text {pico }}\right)$. La producción máxima $\left(Y_{\max }\right)$ o producción mínima $\left(\mathrm{Y}_{\min }\right)$, esta última en el caso de los porcentajes de grasa y proteína, se obtuvo reemplazando el tiempo al pico en la fórmula inicial.

Análisis estadístico. Para las variables, $\beta_{0}, T_{\text {pico }}$ y $Y_{\max } \circ Y_{\min }$ se determinó el efecto del hato, el número de parto y la época y año de parto. Se empleó el procedimiento 
GLM del paquete estadístico SAS $($ (9), mediante el siguiente modelo:

$$
y_{i j k l m n}=\mu+\alpha_{i}+\delta_{j}+\theta_{k}+\varphi_{l}+\gamma_{m(l)}+\varepsilon_{n(i j k l m)}
$$

Donde:

$Y_{i j k l m n}$ es la variable respuesta $\left(\beta_{0}, T_{\text {pico }}\right.$ y $Y_{\text {max }}$ ó $Y_{\text {min }}$ ) en las variables producción de leche y porcentajes de grasa y proteína; $\mu$ es la media general; $a_{i}, \delta_{j}, \theta_{k}, \varphi_{1}$ son los efectos fijos de número de parto ( $i=1,2, \ldots, \geq 6)$, época de parto $(j=1,2, \ldots, 4)$, año de parto $(k=2007,2008$ y 2009) y hato $(I=1,2, \ldots$, 19), respectivamente; y $Y_{m(I)}$ y $\varepsilon_{n(i j k l m)}$ son los efectos aleatorios de animal anidado en hato y el error asociado a cada observación, respectivamente.

\section{RESULTADOS}

En las variables porcentaje de grasa y proteína el modelo de Wood presentó el PAIC, PBIC y $\mathrm{PR}^{2}$ más altos.

Para la producción de leche el modelo de Wilmink presentó el PAIC y PBIC más alto, sin embargo presentó el $\mathrm{PR}^{2}$ más bajo de los cuatro modelos (Tabla 2).

Tabla 2. Criterios de comparación para escoger el mejor modelo que ajuste la curva de producción de leche y los porcentajes de grasa y proteína de vacas Holstein en Antioquia.

\begin{tabular}{|c|c|c|c|c|c|c|c|}
\hline Variable & Modelo & PCCON & PCSIG & PAIC & PBIC & $\mathbf{R}^{2}$ & $\mathbf{P R}^{2}$ \\
\hline \multirow{4}{*}{$\begin{array}{l}\text { Producción } \\
\text { de leche }\end{array}$} & Wood & 65.15 & 65.95 & 40.23 & 36.34 & 97.37 & 58.24 \\
\hline & Brody & 17.29 & 89.61 & 5.90 & 5.23 & 93.96 & 17.43 \\
\hline & Wilmink & 99.39 & 68.63 & 43.02 & 48.18 & 76.66 & 1.74 \\
\hline & $\begin{array}{l}\text { Papajcsik } \\
\text { y Bodero }\end{array}$ & 97.39 & 99.06 & 10.85 & 10.25 & 91.95 & 22.59 \\
\hline \multirow{4}{*}{$\begin{array}{c}\text { Porcentaje } \\
\text { de grasa }\end{array}$} & Wood & 93.22 & 97.25 & 49.97 & 49.83 & 98.04 & 57.22 \\
\hline & Brody & 57.02 & 97.45 & 35.66 & 35.73 & 98.82 & 37.00 \\
\hline & Wilmink & 100 & 39.29 & 3.96 & 4.37 & 55.60 & 0.74 \\
\hline & $\begin{array}{l}\text { Papajcsik } \\
\text { y Bodero }\end{array}$ & 100 & 99.73 & 10.41 & 10.07 & 94.61 & 5.04 \\
\hline \multirow{4}{*}{$\begin{array}{l}\text { Porcentaje } \\
\text { de proteína }\end{array}$} & Wood & 94.90 & 99.19 & 64.67 & 64.73 & 99.18 & 66.62 \\
\hline & Brody & 63.33 & 98.52 & 29.15 & 29.15 & 99.31 & 29.62 \\
\hline & Wilmink & 100 & 22.83 & 1.95 & 2.22 & 45.53 & 0.54 \\
\hline & $\begin{array}{l}\text { Papajcsik } \\
\text { y Bodero }\end{array}$ & 100 & 99.87 & 4.23 & 3.90 & 95.15 & 3.22 \\
\hline
\end{tabular}

Porcentaje de curvas que convergieron; PCSIG: Porcentaje de curvas significativas $(p<0.05)$; PAIC: Porcentaje de curvas con el valor más bajo de AIC; PBIC: Porcentaje de curvas con el valor más bajo de BIC; $\mathrm{R}^{2}$ : Coeficiente de determinación y $\mathrm{PR}^{2}$ : Porcentaje de curvas con el valor más alto de $\mathrm{R}^{2}$.
En las figuras 1,2 y 3 se muestran las curvas de lactancia para producción de leche y para los porcentajes de grasa y proteína estimadas en cada uno de los partos.

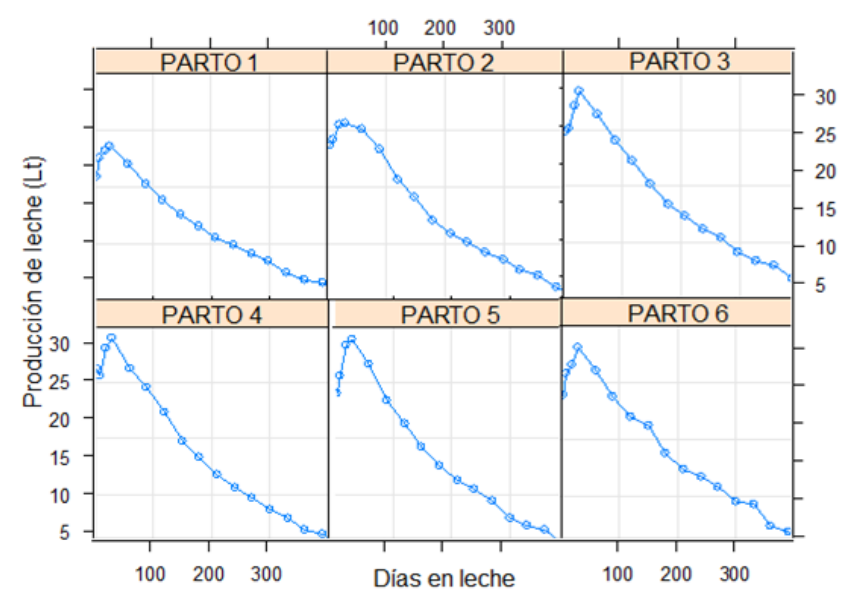

Figura 1. Curvas de lactancia según el modelo de Wood para producción de leche de vacas Holstein en Antioquia.

Las mayores producciones de leche fueron alcanzadas en los partos 3, 4 y 5 , obteniendo las mayores producciones iniciales y al pico de producción. El parto 1 obtuvo las menores producciones durante toda la lactancia (Figura 1).

En el porcentaje de grasa, los valores iniciales más altos fueron en los partos 5 y 6 , en el porcentaje de proteína fueron en los partos 2 y 5 . Las curvas de lactancia para porcentaje de proteína, a diferencia de las curvas para porcentaje de grasa, se caracterizaron por presentar después del pico mínimo de producción una producción muy constante durante toda la lactancia. Por el contrario, los valores porcentuales de la grasa incrementaron hacia el final de la lactancia (Figuras 2 y 3 ).

Los estimativos para las derivadas de las curvas de lactancia del modelo de Wood se encuentran en la tabla 3. La variable días al pico $\left(t_{\text {pico }}\right)$ no presentó diferencias significativas $(p>0.05)$ entre cada uno de los partos y años de parto en las características producción de leche y porcentaje de grasa y proteína (Tabla 3).

Para la producción de leche las variables $\beta_{0}$ y $Y_{\max }$ presentaron distribuciones muy 


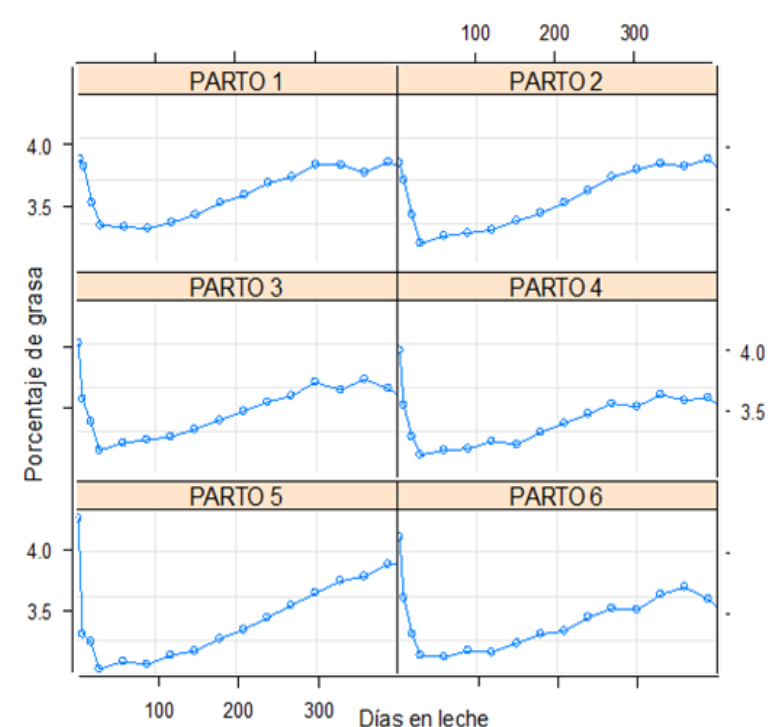

Figura 2. Curvas de lactancia según el modelo de Wood para porcentaje de grasa de vacas Holstein en Antioquia.

similares, donde los mayores valores se encontraron en el parto 3 y el menor valor en el parto 1 con diferencia significativa $(p<0.05)$. Caso contrario se observa en los porcentajes de grasa y proteína, donde para las variables $\beta_{0}$ y $Y_{\max }$ los mayores valores se observan en animales de primer parto y los menores valores en animales de tercer parto (Tabla 3).

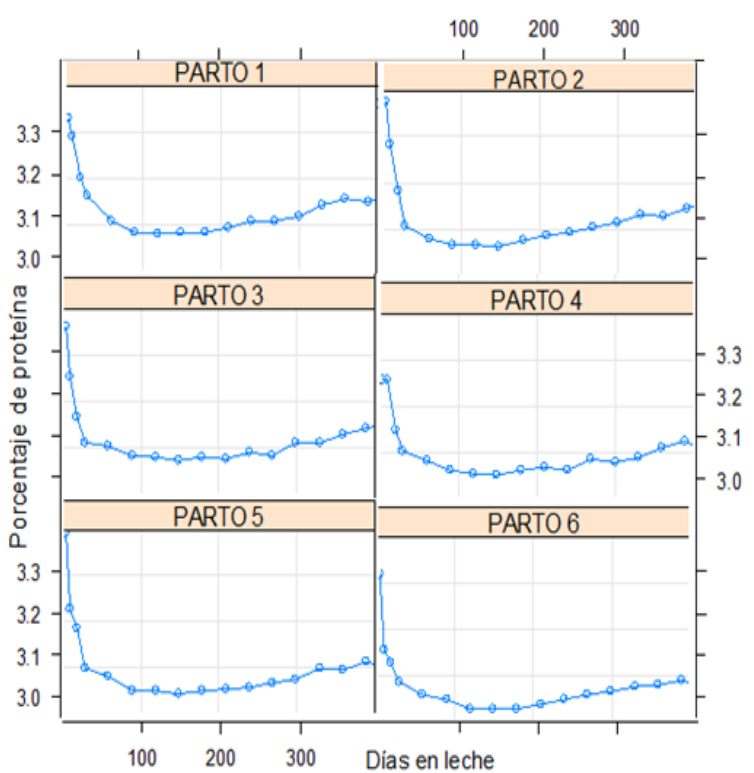

Figura 3. Curvas de lactancia según el modelo de Wood para porcentaje de proteína de vacas Holstein en Antioquia.

Las características $\beta_{0}$ y $Y_{\max }$ han disminuido en el transcurso de los años pero sin diferencia significativa $(p>0.05)$, en los porcentajes de grasa y proteína $\beta_{0}$ y $Y_{\max }$ han aumentado presentando inclusive diferencias significativas $(p<0.05)$ entre los años 2007 y 2009 (Tabla 3).

Tabla 3. Medias y Desviaciones Estándar de variables asociadas a la producción de leche estimadas en seis partos y en tres años de partos en vacas Holstein de Antioquia.

\begin{tabular}{|c|c|c|c|c|c|c|c|c|c|}
\hline & \multicolumn{3}{|c|}{ Producción de Leche } & \multicolumn{3}{|c|}{ Porcentaje de Grasa } & \multicolumn{3}{|c|}{ Porcentaje de Proteína } \\
\hline & $\beta_{0}$ & $\mathrm{t}_{\text {pico }}$ & $Y_{\max }$ & $\beta_{0}$ & $\mathrm{t}_{\text {pico }}$ & $Y_{\min }$ & $\beta_{0}$ & $t_{\text {pico }}$ & $Y_{\min }$ \\
\hline & Media \pm SD & Media \pm SD & Media \pm SD & Media \pm SD & Media \pm SD & Media \pm SD & Media \pm SD & Media \pm SD & Media \pm SD \\
\hline \multicolumn{10}{|l|}{ Parto } \\
\hline 1 & $16.8 c \pm 0.60$ & $35.5 a \pm 1.55$ & $27.4 c \pm 0.50$ & $4.79 a \pm 0.18$ & $71.9 a \pm 2.87$ & $3.13 a \pm 0.04$ & $3.40 \mathrm{a} \pm 0.07$ & $87.0 \mathrm{a} \pm 3.21$ & $3.04 a \pm 0.01$ \\
\hline 2 & $19.3 b \pm 0.55$ & $29.3 a \pm 1.42$ & $31.9 b \pm 0.46$ & $4.66 a \pm 0.17$ & $70.0 \mathrm{a} \pm 2.78$ & $3.04 a b \pm 0.03$ & $3.31 a \pm 0.07$ & $85.9 a \pm 3.15$ & $3.02 \mathrm{a} \pm 0.01$ \\
\hline 3 & $20.0 \mathrm{a} \pm 0.60$ & $28.1 \mathrm{a} \pm 1.55$ & $33.3 a \pm 0.49$ & $4.54 b \pm 0.19$ & $69.6 a \pm 3.09$ & $2.98 b \pm 0.04$ & $3.25 a \pm 0.08$ & $84.3 a \pm 3.43$ & $3.02 \mathrm{a} \pm 0.02$ \\
\hline 4 & $19.9 a \pm 0.67$ & $31.4 \mathrm{a} \pm 1.73$ & $33.1 \mathrm{a} \pm 0.56$ & $4.61 a b \pm 0.21$ & $63.2 \mathrm{a} \pm 3.37$ & $3.02 b \pm 0.05$ & $3.27 a \pm 0.09$ & $84.2 \mathrm{a} \pm 3.74$ & $3.00 \mathrm{a} \pm 0.02$ \\
\hline 5 & $19.1 b \pm 0.76$ & $29.7 a \pm 1.96$ & $33.1 \mathrm{a} \pm 0.63$ & $4.70 \mathrm{a} \pm 0.22$ & $73.4 a \pm 3.54$ & $3.07 a b \pm 0.05$ & $3.21 a \pm 0.09$ & $86.1 \mathrm{a} \pm 4.15$ & $3.00 a \pm 0.02$ \\
\hline$\geq 6$ & $18.9 b \pm 0.75$ & $32.7 a \pm 1.95$ & $32.4 b \pm 0.62$ & $4.90 \mathrm{a} \pm 0.22$ & $70.4 a \pm 3.53$ & $3.21 \mathrm{a} \pm 0.05$ & $3.39 a \pm 0.09$ & $86.9 a \pm 3.74$ & $3.03 a \pm 0.03$ \\
\hline \multicolumn{10}{|c|}{ Año de Parto } \\
\hline 2007 & $20.3 a \pm 0.60$ & $32.9 a \pm 1.56$ & $32.5 a \pm 0.50$ & $4.42 b \pm 0.15$ & $72.8 a \pm 4.41$ & $2.91 b \pm 0.06$ & $3.09 b \pm 0.12$ & $85.1 a \pm 5.23$ & $2.95 b \pm 0.02$ \\
\hline 2008 & $19.4 a \pm 0.38$ & $31.1 \mathrm{a} \pm 0.97$ & $31.5 a \pm 0.31$ & $4.63 a b \pm 0.11$ & $68.9 a \pm 1.84$ & $3.10 a b \pm 0.02$ & $3.20 a b \pm 0.05$ & $82.6 a \pm 1.99$ & $3.02 a b \pm 0.01$ \\
\hline 2009 & $19.1 \mathrm{a} \pm 0.52$ & $29.5 a \pm 1.49$ & $31.0 \mathrm{a} \pm 0.48$ & $4.89 a \pm 0.27$ & $67.6 a \pm 2.46$ & $3.15 a \pm 0.03$ & $3.34 \mathrm{a} \pm 0.06$ & $80.6 a \pm 2.80$ & $3.06 a \pm 0.01$ \\
\hline
\end{tabular}

Letras diferentes en la misma columna indican diferencia significativa según la prueba de Tukey - Kramer $(p \leq 0.05)$. $\beta_{0}=$ Coeficiente que está relacionado con la producción inicial de leche o con el porcentaje inicial de grasa y proteína (modelo de Wood). $t_{\text {pico }}=$ Día en la cual se alcanza el pico de producción máximo (producción de leche) o mínimo (porcentaje de grasa y proteína) (modelo de Wood) $Y_{\max } \circ Y_{\min }=$ Producción de leche en el pico máximo o porcentaje de grasa y proteína en el pico mínimo de lactancia (modelo de Wood). 


\section{DISCUSIÓN}

Los valores de PAIC, PBIC y $\mathrm{PR}^{2}$ encontrados en el modelo de Wood para las variables porcentaje de grasa y proteína indican que es el modelo más apropiado para modelar curvas de lactancia en grasa y proteína. En la producción de leche, pese a que el modelo de Wilmink presentó los valores más altos de PAIC y PBIC, no es el modelo más apropiado debido al bajo valor que presentó de $\mathrm{PR}^{2}$, siendo este el más bajo de los cuatro modelos (Tabla 2).

Para grasa y proteína, Quinn et al (10) compararon entre los modelos de Wood, Wilmink y Guo \& Swalve, el modelo de Wilmink obtuvo mejor bondad de ajuste y capacidad para predecir la producción total de grasa y proteína. Estos resultados difieren a los encontrados en este trabajo, donde el modelo de Wilmink presentó los peores ajustes (Tabla 2). Esto puede explicarse debido a que el modelo de Wilmink, en comparación con el modelo de Wood, no presenta buenos ajustes cuando la curva tiene una forma opuesta a la de producción de leche; además tiende a sobrestimar la producción al final de la lactancia, especialmente en los porcentajes de proteína $(2,10)$.

Las gráficas de producción de leche en comparación con las gráficas de porcentaje de grasa muestran una relación inversa, donde los mayores valores de producción coinciden con los menores valores de porcentaje de grasa. Gráficas similares fueron encontradas por Silvestre et al (7) y Quinn et al (10), quienes mostraron esta relación inversa entre la producción de leche y los porcentajes de grasa y proteína. Sin embargo, estos autores muestran una gráfica de porcentaje de proteína que incrementa sus valores hacia el final de la lactancia, resultados que difieren a los obtenidos en este trabajo, donde el porcentaje de proteína después del pico de lactancia permanece constante (Figuras 1, 2 y 3$)$.

Esta premisa es debida a que el porcentaje de proteína no se ve fuertemente afectado por cambios en la dieta, ni por efectos de dilución cuando la producción de leche disminuye al finalizar la lactancia.

Según Silvestre et al (7), en los porcentajes de grasa y proteína, el punto más bajo $\left(\mathrm{Y}_{\min }\right)$ aumenta del primer al segundo parto y permanece constante durante los demás partos. En este trabajo se observó que la proteína permanece constante entre los diferentes partos pero en el caso de la grasa se observaron diferencias significativas entre los partos (Tabla 3).

A medida que aumenta la edad o el número de lactaciones aumenta la producción de leche y desciende los porcentajes de grasa y sólidos totales. A partir de la cuarta lactación los porcentajes de los componentes sólidos vuelven a incrementarse, principalmente en el porcentaje de grasa, el contenido proteico apenas se modifica $(7,10,11)$.

Rodríguez et al (11), analizando producción de leche en ganado Holstein encontraron que hembras de primer parto presentan un pico de producción bajo comparadas con hembras multíparas. Resultados similares a los obtenidos en este trabajo (Tabla 3).

En conclusión, el modelo de Wood fue el modelo que mejor explicó las curvas de lactancia para producción de leche y para los porcentajes de grasa y proteína. En este modelo los factores de número y año de parto fueron los más influyentes en cada uno de los parámetros estimados. La curva de lactancia para el porcentaje de proteína se caracterizó por ser muy consistente y no presentar mayores fluctuaciones durante toda la lactancia.

\section{Agradecimientos}

Los autores agradecen al Ministerio de Agricultura y Desarrollo Rural y al Fondo Nacional del Ganado por la financiación de este trabajo e igualmente al apoyo dado por la Corporación Antioquia Holstein y de cada una de las haciendas en la elaboración del control lechero. Este artículo hace parte del proyecto de grado de maestría en Ciencias Animales del primer autor. 


\section{REFERENCIAS}

1. Pérez $\mathrm{PL}$, Anrique $\mathrm{GR}$, González VH. Factores no genéticos que afectan la producción y composición de la leche en un rebaño de pariciones biestacionales en la décima región de los lagos, Chile. Agr Tec Chile 2007; 67(1):39-48.

2. Quintero JC, Serna J, Hurtado N, Rosero $\mathrm{R}$, Cerón M. Modelos matemáticos para curvas de lactancia en ganado lechero. Rev Col Cienc Pec 2007; 20:149-156.

3. García SC, Holmes CW. Lactation curves of autumn- and spring-calved cows in a pasture-based dairy system. Livest Prod Sci 2001; 68:189-203.

4. Wood PDP. Algebraic model of the lactation curve in cattle. Nature 1967; 216:164-165.

5. Wilmink JBM. Comparison of different methods of predicting 305 - day milk yield using means calculated from within herd lactation curves. Livest Prod Sci 1987; 17:1-17.

6. Brody S, Ragsdale AC, Turner CW. The relation between the initial rise and the subsecuent decline of milk secretion following parturition. J Gen Physiol 1924; 6:541-545.
7. Silvestre AM, Martins AM, Santos VA, Ginja MM, Colaço JA. Lactation curves for milk, fat and protein in dairy cows: A full approach. Livest Sci 2009; 122: 308-313.

8. Papajcsik IA, Bodero J. Modeling lactation curves of Friesian cows in a subtropical climate. Anim Prod 1988; 47:201-207.

9. SAS/STAT: Guide for Personal Computer [programa de ordenador]. Versión 9.1 Cary (NC): SAS Institute Incorporation; 2006.

10. Quinn N, Killen L, Buckley F. Modelling fat and protein concentration curves for Irish dairy cows. Irish J Agr Food Res 2006; 45:13-23.

11. Rodríguez ZL, Ara GM, Huamán UH, Echevarría CL. Modelos de ajuste para curvas de lactación de vacas en crianza intensiva en la cuenca de Lima. Rev Inv Vet Perú 2005; 16(1):1-12. 